Meta

Journal des traducteurs

Translators' Journal

\title{
Esquisse d'une nouvelle microstructure de dictionnaire spécialisé reflétant la variation en discours du terme syntagmatique
}

\section{Tanja Collet}

Volume 49, numéro 2, juin 2004

URI : https://id.erudit.org/iderudit/009349ar

DOI : https://doi.org/10.7202/009349ar

Aller au sommaire du numéro

Éditeur(s)

Les Presses de l'Université de Montréal

ISSN

0026-0452 (imprimé)

1492-1421 (numérique)

Découvrir la revue

Citer cet article

Collet, T. (2004). Esquisse d'une nouvelle microstructure de dictionnaire spécialisé reflétant la variation en discours du terme syntagmatique. Meta, 49(2), 247-263. https://doi.org/10.7202/009349ar

\section{Résumé de l'article}

Cet article s'attache à présenter une nouvelle microstructure qui se distancie du primat traditionnellement accordé à la dimension notionnelle du terme. De nature sémasiologique, cette microstructure s'intéresse, en effet, davantage à la représentation des caractéristiques lexico-syntaxiques des rapports du terme, spécialement du terme syntagmatique, au texte, tout en fournissant les renseignements notionnels qui s'imposent. L'article se penchera d'abord sur la mise en discours du terme et montrera comment celui-ci participe, par le biais de variantes formelles engendrées en discours par des opérations syntaxiques, à l'organisation de la cohésion du texte de spécialité. Il proposera ensuite une microstructure qui cherche à informer sur cet aspect du comportement discursif du terme, spécialement du terme syntagmatique. 


\title{
Esquisse d'une nouvelle microstructure de dictionnaire spécialisé reflétant la variation en discours du terme syntagmatique ${ }^{\prime}$
}

\author{
TANJA COLLET \\ University of Windsor, Windsor, Canada \\ tcollet@uwindsor.ca
}

\section{RÉSUMÉ}

Cet article s'attache à présenter une nouvelle microstructure qui se distancie du primat traditionnellement accordé à la dimension notionnelle du terme. De nature sémasiologique, cette microstructure s'intéresse, en effet, davantage à la représentation des caractéristiques lexico-syntaxiques des rapports du terme, spécialement du terme syntagmatique, au texte, tout en fournissant les renseignements notionnels qui s'imposent. L'article se penchera d'abord sur la mise en discours du terme et montrera comment celui-ci participe, par le biais de variantes formelles engendrées en discours par des opérations syntaxiques, à l'organisation de la cohésion du texte de spécialité. Il proposera ensuite une microstructure qui cherche à informer sur cet aspect du comportement discursif $d u$ terme, spécialement du terme syntagmatique.

\begin{abstract}
The purpose of this article is to present a new microstructure which attempts to break away from the traditional predominance of the conceptual dimension of the headword in specialized dictionary entries. Proceeding semasiologically, the microstructure provides not only the conceptual information dictionary users are typically interested in, but also detailed information about the lexico-syntactic characteristics of the relationships holding between terms, particularly compound terms, and the specialized texts that contain them. The article focuses first on the behavioural patterns of terms in discourse and particularly on how terms, and their variants generated by syntactic operations in discourse, augment the overall cohesion of a specialized text. It then introduces the reader to the new microstructure which incorporates information about this particular behavioural pattern.
\end{abstract}

\section{MOTS-CLÉS/KEYWORDS}

dictionnaire spécialisé monolingue, microstructure, terme syntagmatique, variation, réduction

\section{Introduction}

C'est un fait bien connu que les consommateurs privilégiés de terminologies (Gouadec 1990: 52) et de ce fait de dictionnaires spécialisés ${ }^{2}$ ne sont guère les professionnels, qui maîtrisent le plus souvent la terminologie de leur domaine, ni les professionnels en devenir qui s'initient à un domaine, mais les traducteurs et rédacteurs techniques, qui assurent la traduction ou la rédaction de textes techniques portant sur des sujets divers.

On sait que les traducteurs et rédacteurs consultent les dictionnaires spécialisés dans le but de se renseigner sur la terminologie ayant cours dans un domaine ou afin 
d'acquérir un minimum de connaissances encyclopédiques ou conceptuelles pertinentes. Les dictionnaires spécialisés se contentant, le plus souvent, de fournir des renseignements de type conceptuel sous la forme d'une définition du terme-vedette - renseignements auxquels s'ajoutent parfois des renseignements de type linguistique ayant trait, par exemple, aux synonymes du terme-vedette - ces ouvrages répondent bien, en règle générale, à ce besoin encyclopédique des traducteurs et rédacteurs techniques. Cependant, les besoins de ces utilisateurs de dictionnaires spécialisés ne se limitent point à la seule acquisition de connaissances encyclopédiques. En effet, les besoins des traducteurs et rédacteurs, qui «opèrent en discours plutôt qu'en langue » (Dancette 1995: 174), sont le plus souvent également discursifs de nature en ce sens qu'ils concernent, en outre, la conception, c'est-à-dire l'écriture, du texte technique. Ainsi, les traducteurs et rédacteurs sont susceptibles de s'interroger sur les façons dont les termes d'un domaine se combinent en phrases et participent à la composition du texte technique. Du fait, cependant, que les dictionnaires spécialisés se consacrent surtout à la définition du terme-vedette et s'intéressent peu, en règle générale, à l'usage de ce dernier, ils ne sont que rarement en mesure de combler ces besoins discursifs de leurs utilisateurs principaux.

À cet égard, une enquête menée par le Centre de Terminologie de Bruxelles auprès d'utilisateurs (traducteurs, rédacteurs, documentalistes et experts) de dictionnaires spécialisés ${ }^{3}$ a révélé que la rareté d’informations portant sur la mise en discours des termes est ressentie comme problématique par nombre d'utilisateurs. Aux dires de Hermans et De Schaetzen (1991: 301), l'enquête aurait même permis « d'affirmer qu'un grand nombre de dictionnaires ne répondent que partiellement, voire très peu, aux besoins et aux souhaits des utilisateurs ». Parmi les faits déplorés par les utilisateurs sur le plan qualitatif, l'enquête aurait identifié entre autres la «rareté des informations [...] phraséologiques, stylistiques [...] pourtant indispensables à l'utilisation adéquate des termes » (Hermans et De Schaetzen 1991:301). Cependant, aux informations phraséologiques et stylistiques réclamées par les consommateurs de dictionnaires, nous croyons qu'il importe d'en ajouter d'autres encore négligées aujourd'hui mais qui se rapportent également à l'emploi du terme dans le texte technique.

En effet, comme tout texte, le texte technique ou de spécialité est un construit faisant preuve de caractéristiques qui décident de sa textualité, c'est-à-dire de son caractère unitaire de texte, telle une certaine cohésion. Comme tout texte, le texte technique se constitue à partir d'un ensemble de mots lexicaux (nom, adjectif, verbe, etc.) et grammaticaux (articles définis et indéfinis, pronoms personnels, adjectifs démonstratifs, etc.) qui construisent un réseau de rapports anaphoriques et cataphoriques formant l'échafaudage qui le soutient. Les termes, qui se rangent dans la catégorie des mots lexicaux, ne sont aucunement exclus de cet échafaudage cohésif, mais participent bien au contraire à sa construction avec les autres mots, grammaticaux ou lexicaux, figurant dans le texte technique. Les termes syntagmatiques, spécialement, jouent un rôle à la fois important et particulier dans la création d'un tel réseau cohésif de rapports anaphoriques et cataphoriques.

Le terme syntagmatique, en effet, qui doit son nom au fait qu'il se constitue d'une suite de constituants (terminal mobile terrestre, satellite stabilisé selon trois axes, etc.), se caractérise généralement par un faible degré de figement, si bien qu’il ne résiste guère aux forces cohésives qui s'exercent sur lui dans le texte de spécialité. Il s'ensuit qu'un tel type de terme se voit souvent transformer, dans le texte de spécialité, par un 
certain nombre d'opérations syntaxiques qui visent à adapter sa linéarité aux caractéristiques de la phrase ou de la suite de phrases qui le contiennent. Parmi ces opérations figure la réduction qui supprime, en fonction des caractéristiques de l'environnement phrastique du terme, un ou plusieurs constituants de celui-ci. Par exemple, si son environnement phrastique l'exige, un terme, tel terminal mobile terrestre, pourra se voir réduire à mobile terrestre, terminal mobile, mobile ou terminal. À la suite d'une telle transformation, une relation cohésive anaphorique ou cataphorique s'érige en général entre le terme, présent dans le texte, et sa variante réduite.

Peu de dictionnaires spécialisés - à notre connaissance aucun - possèdent une microstructure qui reflète cette souplesse du terme syntagmatique, c'est-à-dire cette capacité du terme syntagmatique de permettre à une opération comme la réduction de dériver de sa linéarité de départ une autre qui est davantage en harmonie avec la suite du texte. Ceci est regrettable, car ces métamorphoses, qui correspondent à une dynamique textuelle, sont susceptibles d'intéresser les consommateurs principaux de ces ouvrages de référence, notamment les traducteurs et rédacteurs techniques.

Nous sommes donc d'accord avec Pearson (1998: 71) qui estime que:

The main drawback of [monolingual specialized] dictionaries is that they do not contain grammatical or phraseological information which means that the dictionary users are given no advice regarding, for example, collocation restrictions on technical terms. Ideally, a specialized dictionary should contain not only all of the information which is already being provided in such dictionaries but also grammatical and phraseological information which would show the user how to use the term correctly.

Toutefois, aux renseignements linguistiques qui, d'après Pearson (1998), devraient figurer dans une entrée de dictionnaire spécialisé, nous jugeons, comme nous l'avons suggéré ci-dessus, qu'il conviendrait d'ajouter d'autres renseignements tout aussi pertinents, tel le paradigme réductionnel du terme syntagmatique, c'est-à-dire les variantes du terme issues d'une opération de réduction. L'inclusion du paradigme réductionnel ne saura être qu'à l'avantage des dictionnaires spécialisés. En effet, cela permettra à ces ouvrages de référence de faire pour ainsi dire d'une pierre deux coups. D’une part, les dictionnaires représenteront un aspect essentiel des rapports du terme au texte, à savoir que le terme contribue à la cohésion du texte de spécialité en se transformant en son sein. D'autre part, les dictionnaires cesseront de perpétuer le mythe, particulièrement malheureux pour ceux qui opèrent en discours, du terme monolithique, immuable en discours d'une occurrence à l'autre.

Étant donné ce qui précède, le but de notre article est de présenter l'ébauche d'une microstructure de dictionnaire qui comporte, outre les rubriques traditionnelles consacrées, par exemple, à la définition et plus rarement aux synonymes du terme-vedette, une rubrique supplémentaire qui représente le paradigme réductionnel du terme-vedette, c'est-à-dire ses linéarités créées par une opération de réduction. Du point de vue théorique, nous nous alignons dans le cadre de cet article sur Slodzian (1997: 29), car nous estimons comme cette terminologue que ce n'est qu'en réservant une place plus importante au syntagmatique et au textuel qu'on «se donnera les moyens de créer des dictionnaires spécialisés plus opérationnels pour les spécialistes qui lisent, écrivent, traduisent des textes scientifiques».

Quant à l'organisation de notre article, celui-ci comportera deux parties. Dans la première, nous décrirons la réduction du terme syntagmatique et démontrerons 
l'importance de cette transformation pour la cohésion du texte de spécialité. Nous nous servirons d'extraits de textes réels pour illustrer les notions introduites. Dans la deuxième, nous esquisserons la microstructure proposée et montrerons comment les métamorphoses observées dans les extraits analysés dans la première partie sont évoquées par elle.

\section{Terme syntagmatique, réduction et cohésion}

Dans le texte de spécialité, le terme syntagmatique représente, cela va sans dire, une notion, mais constitue également un moyen cohésif. En effet, il établit des relations de sens au sein de la phrase, entre ses parties constituantes, et au delà de la phrase, entre les phrases constituantes du texte. Le terme syntagmatique parvient à tisser de telles relations entre autres grâce à une opération syntaxique, nommée "réduction », qui adapte sa linéarité aux exigences de son environnement phrastique, quand cet environnement est d'une certaine nature et nécessite entre autres choses la réitération du terme syntagmatique. Dans ce sous-point, nous allons définir la réduction, distinguer ses produits de la néonymie et de la synonymie et enfin montrer comment cette opération syntaxique permet au terme syntagmatique d'agir comme un moyen cohésif. Les notions abordées dans ce sous-point nous permettront de renseigner, par ailleurs, sur quelques aspects de la microstructure de dictionnaire qui fera l'objet du sous-point 3 , car l'architecture de cette microstructure intègre certaines de ces notions.

\subsection{Réduction: définition}

La réduction peut être définie comme une opération syntaxique à la fois intrasyntagmatique et intratextuelle qui transforme globalement un terme syntagmatique en supprimant un ou plusieurs de ses constituants et cela sans modifier tant soit peu son sens ${ }^{4}$. Il nous semble important d'expliquer sommairement les composantes principales de cette définition qui sont au nombre de trois, à savoir (a) mécanisme intrasyntagmatique, (b) mécanisme intratextuel et (c) maintien du sens du terme syntagmatique.

La réduction est un mécanisme intrasyntagmatique. Elle ne transforme que des unités faiblement figées de type syntagmatique, mais son résultat peut être graphiquement simple, c'est-à-dire non syntagmatique. Nous appelons «paire réductionnelle» deux formes d'un même terme syntagmatique présentes dans un même texte et liées par un rapport réductionnel, en ce sens que l'une des deux formes constitue une réduction de l'autre. Pour la représenter, nous mettons les deux formes entre parenthèses, comme suit: $\{X, Y\}$, où $X$, le premier membre de la paire, correspond au terme qui subit la réduction, et $\mathrm{Y}$, le deuxième membre, à son résultat. Sur le plan de leur linéarité, $\mathrm{X}$ et $\mathrm{Y}$ doivent respecter deux conditions évidentes : $\mathrm{X}$ doit compter plus de constituants que $\mathrm{Y}$ et tous les constituants de $\mathrm{Y}$ doivent être contenus dans $\mathrm{X}$.

La réduction est, en outre, un mécanisme intratextuel. Elle répond, en effet, à des déclencheurs purement textuels, dont le plus caractéristique est la réitération du terme syntagmatique, c'est-à-dire la répétition de ce syntagme au sein d'une même phrase ou d'une phrase à l'autre au sein d'un même texte. Une telle répétition a le plus souvent pour conséquence malencontreuse d'éroder le contenu informatif du terme à cet endroit précis du texte. De Beaugrande et Dressler (1986: 57), qui estiment que 
la réitération "has the disadvantage [...] of reducing informativity», constatent que pour atténuer cette érosion de l'information véhiculée par une unité «techniques are often used in which forms recur with somewhat different content, or content recurs with different forms (c'est nous qui soulignons) ». Nous en concluons qu'il existe, selon toute probabilité, un lien de cause à effet entre la réitération du terme syntagmatique et ses métamorphoses discursives par réduction, la transformation de la linéarité du terme par l'effacement de quelques-uns de ses constituants faisant sans doute contrepoids à la baisse du contenu informatif du terme occasionnée par sa réitération. Il nous faut, toutefois, formuler une mise en garde à propos de la réitération, notamment qu'il serait faux d'en déduire que la forme réduite suit dans tous les cas la forme pleine du terme - la situation inverse se rencontre également en discours - ou que les suppressions se font de manière graduelle en cas de réductions successives. Quant au rapport réductionnel, créé par la réduction entre le terme syntagmatique et sa variante réduite, il peut être de nature intraphrastique ou interphrastique. Par conséquent, la distance séparant les deux membres d'une paire réductionnelle est variable, et peut aller de quelques mots à quelques phrases.

Enfin, la réduction ne modifie en rien le sens du terme syntagmatique. Bien au contraire, elle le maintient tel quel et prend soin de le transmettre à la variante réduite, de sorte que le terme et sa variante demeurent au moins localement équivalents. À cette fin, elle se sert entre autres d'indices contextuels, qui environnent la paire réductionnelle, particulièrement la variante réduite. Les indices contextuels sont de trois types au moins: (a) la proximité de la forme pleine non réduite du terme, (b) des mots lexicaux ayant un sens identique ou similaire à celui du ou des constituants effacés ou (c) des mots grammaticaux, tels l'adjectif démonstratif et l'article défini, qui véhiculent une présupposition explicite, en ce sens qu'ils renvoient à un antécédent situé ailleurs dans le texte, en l'occurrence la forme non réduite du terme. Les extraits qui suivent nous permettront d'illustrer l'importance de ces indices pour le transfert du sens de la forme pleine à la variante réduite du terme.

Extrait 1:

C'était également l'objet de la Conférence administrative mondiale des Radiocommunications qui s'est tenue en 1985 pour débattre de l'utilisation de l'orbite des satellites géostationnaires et de la planification des services spatiaux utilisant cette orbite ${ }^{5}$ (Brillaud 1988: 570-11).

\section{Extrait 2:}

La masse de carburant nécessaire à cette circularisation est approximativement équivalente à celle du satellite une fois en orbite géostationnaire: ainsi, un satellite de $2000 \mathrm{~kg}$ au lancement ne pèse plus qu'environ $1000 \mathrm{~kg}$ en orbite géostationnaire.

Un satellite mis en orbite basse lors du lancement a besoin, quant à lui, de deux moteurs pour rejoindre l'orbite des satellites géostationnaires, un moteur de périgée pour le placer sur une orbite de transfert et un moteur d'apogée (Brillaud 1988: 570-2).

\section{Extrait 3:}

Étant donné que la f.e.m. d'un élément de batterie est de l'ordre du volt, comme pour les cellules solaires, il sera nécessaire d'assembler plusieurs éléments pour constituer une batterie. [...] Exemples:- Une batterie de 15 éléments $\mathrm{Ni}-\mathrm{Cd}$ de $6 \mathrm{Ah}$ pèse $6,2 \mathrm{~kg}$ [...] (Fitamant 1971:29). 
Le premier extrait, ou contexte réductionnel, contient la paire réductionnelle \{orbite des satellites géostationnaires, orbite\}. On constate que la réduction est intraphrastique dans ce contexte réductionnel, que la forme réduite suit la forme pleine du terme syntagmatique, et que l'adjectif démonstratif, qui la précède, témoigne de sa coréférentialité avec cette forme pleine. En effet, l'adjectif démonstratif cette, qui véhicule une présupposition explicite, rattache orbite à son antécédent, c'est-à-dire à une unité de sens précis déjà employée à un autre endroit dans le texte et qui permet sa désambiguïsation. Cet antécédent est orbite des satellites géostationnaires, le terme syntagmatique transformé par la réduction en l'unité simple, orbite. Bref, dans ce contexte réductionnel, la réduction exploite la proximité des deux membres de la paire réductionnelle et utilise l'adjectif démonstratif afin d'assurer la transition du sens de la forme non réduite du terme à la variante réduite, soit de orbite des satellites géostationnaires à orbite.

Quant au deuxième extrait, du même texte, il renferme la paire \{orbite des satellites géostationnaires, orbite géostationnaire\}. On constate que la réduction est interphrastique cette fois-ci, car elle fait intervenir deux phrases, et que la forme réduite précède la forme pleine du terme syntagmatique. Outre un indice contextuel, notamment la proximité des deux membres de la paire réductionnelle, la coréférentialité de la forme pleine et de la forme réduite est mise en évidence, dans ce contexte, par un constituant que les deux formes ont en commun, notamment géostationnaire. Ce constituant confirme que la forme réduite désigne une même sorte d'orbite que la forme pleine du terme réitéré, notamment une orbite de satellite, située dans le plan de l'équateur à une distance de $36000 \mathrm{~km}$ au-dessus de celui-ci, sur laquelle un satellite se déplace à une vitesse égale à la vitesse de rotation de la Terre, de sorte qu'il apparaît immobile pour tout point de la Terre.

Il ressort des deux premiers extraits examinés qu'un même terme syntagmatique, par exemple orbite des satellites géostationnaires, peut faire l'objet de plusieurs réductions au sein d'un même texte, et que ces réductions assurent à l'intérieur du contexte réductionnel la coréférentialité des formes pleine et réduites du terme qu'elles transforment. Cependant, les deux formes réduites, relevées dans ces deux extraits, ne sont pas de même nature. Elles se distinguent par un trait latent, qui concerne la portée de leur coréférentialité avec la forme pleine du terme, c'est-à-dire leur capacité de véhiculer le sens de la forme pleine dans un contexte autre que le contexte réductionnel. De ce point de vue, orbite géostationnaire nous semble investi d'une plus grande autonomie contextuelle que orbite. En effet, en ce sens que orbite ne peut signifier orbite des satellites géostationnaires que dans le contexte réductionnel, étant donné l'existence de plusieurs autres sortes d'orbites. La coréférentialité de orbite avec orbite des satellites géostationnaires repose entièrement sur des indices de type contextuel, tels que la présence d'un adjectif démonstratif. Par conséquent, elle ne se réalise que lorsque orbite et orbite des satellites géostationnaires cooccurrent au sein d'un même contexte régi par un rapport réductionnel. En revanche, le sémantisme de orbite géostationnaire paraît suffisamment fort pour admettre un emploi autonome. La coréférentialité de orbite géostationnaire avec orbite des satellites géostationnaires, quoique tributaire également de la proximité de ces deux formes dans le contexte réductionnel analysé, repose davantage sur la présence d'un constituant, notamment géostationnaire. Par conséquent, elle ne nécessite point en tout temps la cooccurrence de orbite géostationnaire et de orbite des satellites géostationnaires au sein d'un même 
contexte réductionnel. Il s'ensuit que orbite géostationnaire est susceptible de transcender l'environnement contextuel immédiat, car, contrairement à orbite, son emploi post-réductionnel ne semble exiger la remise en évidence du rapport réductionnel. Nous en concluons qu'il convient de distinguer deux types de réductions, qui s'opposent par le degré d'autonomie contextuelle dont jouissent leurs produits. Nous qualifions de réduction contextuelle non lexicogène $(\mathrm{RC})$ le premier type de réduction, qui engendre des paires réductionnelles du genre \{orbite des satellites géostationnaires, orbite\}, et de réduction contextuelle lexicogène $(\mathrm{RL})$, le deuxième. Il va sans dire que la distinction que nous venons d'établir entre réduction contextuelle non lexicogène et réduction contextuelle lexicogène aura des répercussions sur la microstructure de dictionnaire qui aura à renseigner sur cette dynamique du terme-vedette.

Enfin, pour ce qui est du troisième et dernier extrait, il contient la paire \{élément de batterie, élément\}. La réduction est intraphrastique dans ce contexte réductionnel, mais on constate que la forme réduite, élément, est réitérée dans une phrase qui suit celle dans laquelle la réduction a eu lieu. La coréférentialité de élément avec élément de batterie relève dans ce contexte également de la proximité des deux membres de la paire réductionnelle, mais est par ailleurs établie par un autre indice contextuel, notamment la présence d'un mot lexical dans le voisinage immédiat de élément et dont le sens est identique à celui du constituant effacé: batterie. Cet indice contextuel accompagne d'ailleurs les deux occurrences de élément. En somme, dans cet extrait, la réduction exploite deux indices contextuels pour assurer la transition du sens de élément de batterie à élément: la proximité de la forme pleine et de la forme réduite du terme et la présence de batterie avant ou après élément.

\subsection{Réduction: néonymie, synonymie ou variation contextuelle}

Nous estimons qu'il convient de distinguer la réduction à la fois de la néonymie et de la synonymie, phénomènes auxquels la réduction a été associée dans la littérature terminologique et dont le premier concerne l'enrichissement du lexique et le second sa structuration au point de vue sémantique. Deux raisons, au moins, nécessitent cette distinction. La première se rapporte à la nature de la réduction, opération syntaxique qui s'opère dans le texte et qui, bien qu'elle transforme des termes présentant des caractéristiques particulières, tel un figement plutôt faible, relève beaucoup moins du lexical que du textuel, étant donné qu'elle est principalement attribuable à des facteurs textuels. La deuxième concerne la nature du contenu sémantique de la forme réduite. Celle-ci véhicule, comme nous l'avons vu, un sens identique à celui dont est porteuse la forme pleine du terme. Cependant, cette identité de sens est établie dans le texte par des indices textuels ou par une combinaison d'indices textuels et d'indices faisant partie intégrante de la forme réduite, et ne subsiste pas toujours hors du texte. Étant donné ces deux raisons, les formes réduites ne sont à considérer ni comme des néonymes ni comme des synonymes mais bien plutôt comme des variantes formelles du terme, qui se voit transformer par la réduction dans le texte qui le contient. Par variante formelle, nous entendons une forme différente d'un terme engendrée en discours par une transformation, telle la réduction. Cette distinction nette que nous préférons faire entre les résultats de la réduction, d'une part, et la néonymie et la synonymie, d'autre part, n'est pas sans importance pour la microstructure dictionnairique que nous proposerons sous 3 . 
La forme réduite n'est pas un néonyme, bien que maints terminologues, tel Kocourek (1991), aient argumenté le contraire, spécialement pour ce qui est des produits de la réduction contextuelle lexicogène. Ainsi, aux dires de Kocourek (1991: 161 ), ce type de réduction créerait «de nouvelles unités lexicales» par la suppression « [d'] un ou [de] plusieurs mots constitutifs du syntagme». Cependant, en syntagmatique, un néonyme est en règle générale le résultat d'une opération contraire; une opération, en effet, qui ne supprime pas de constituants mais ajoute à une base, qui peut être simple, par exemple station, ou syntagmatique, par exemple station terrienne, un déterminant qui crée entre la base et le néonyme, par exemple entre station et station terrienne ou entre station terrienne et station terrienne côtière, une filiation hyperonymique. En vertu de ce rapport, le néonyme dénomme généralement une notion qui est un hyponyme de celle dénommée par la base. On peut en conclure que la réduction et la néonymie constituent deux phénomènes bien distincts, étant donné que la réduction ne possède, contrairement à la néonymie, aucune propriété véritablement néologique. En effet, la réduction ne dérive pas du terme transformé une dénomination hyponymique nouvelle et tout à fait autonome, mais génère au contraire une forme différente investie du même sens et dotée d'une autonomie variable. Ce caractère différent refuse, à notre avis, à la forme réduite, même lexicogène, le statut de néonyme.

Dans le domaine dictionnairique, le statut non néonymique des produits de la réduction n'est pas sans conséquence. En effet, du fait de ce statut, ces formes, qui sont à considérer comme des variantes formelles d'un terme, ne peuvent être placées à la tête d'une entrée indépendante, séparée de celle de la forme canonique du terme. Il semble plus judicieux, en effet, de regrouper dans une même entrée la forme pleine d'un terme ainsi que ses variantes formelles issues de réductions contextuelles lexicogènes ou non lexicogènes. Quant à l'ordre à donner aux différentes formes d'un terme syntagmatique au sein d'une même entrée, cette question sera traitée sous 3.1.

La forme réduite n'est pas non plus un synonyme, malgré le transfert du sens de la forme pleine à la forme réduite, transfert sémantique qui a conduit plus d'un terminologue à qualifier cette variation de synonymique. Sager (1984: 322), par exemple, qui a le mérite d'être un des premiers terminologues à avoir attiré l'attention sur la variation contextuelle et la réduction, note que dans un même texte «one finds three or four alternative designations for compounds» et que "such cases of synonymic variation are not rare». Cependant, selon nous, il importe de bien distinguer la réduction de la synonymie, vu qu'il s'agit ici aussi de deux phénomènes linguistiques différents, l'un d'ordre textuel et transformationnel et l'autre relevant de la sémantique lexicale, que l'on a tendance à rapprocher seulement parce que les unités, que l'un engendre et que l'autre réunit, présentent la caractéristique de partager leur sens avec d'autres unités. Dans le cas de la réduction, toutefois, ces unités sont des formes différentes, donc des variantes formelles, d'un terme pré-existant, alors que dans celui de la synonymie, il s'agit d'unités autonomes provenant d'étymons différents.

Dans le domaine dictionnairique, le statut non synonymique des formes réduites n'est pas non plus sans conséquence. En effet, n'étant pas des synonymes, il va sans dire que les formes réduites ne peuvent être mentionnées dans la rubrique d'une entrée dictionnairique consacrée aux synonymes du terme-vedette, car il convient d'éviter que cette rubrique ne devienne un fourre-tout contenant des unités de nature dispa- 
rate. D'ailleurs, du fait que tout synonyme, au sens strict du terme, est également susceptible de se voir réduire en discours s'il est de type syntagmatique, un synonyme peut également posséder plusieurs formes, lesquelles devraient être listées dans l'entrée dont il est le terme-vedette.

\subsection{Réduction: source de cohésion}

La réduction du terme syntagmatique en discours possède des propriétés textogènes ${ }^{6}$. En effet, les effacements non lexicogènes \{orbite des satellites géostationnaires, orbite\} et lexicogènes, \{orbite des satellites géostationnaires, orbite géostationnaire\} participent, au moment de la production d'un texte, activité soumise aux forces du principe du moindre effort (Zipf 1965), à l'équilibrage des deux efforts d'encodage et de décodage. L'équilibrage de ces forces, dont le premier se rapporte à celui qui écrit le texte et le deuxième à celui qui le lit, est crucial, car les économies de travail, réalisées lors de l'encodage, ne peuvent en aucun cas compliquer le décodage, un décodage long et pénible risquant, en effet, de se solder par un échec de communication. Favorables à la fois à l'encodage et au décodage, les deux types d'effacement sont fondamentalement textogènes.

Du point de vue de l'encodage, les effacements lexicogènes et non lexicogènes effectués par la réduction comptent parmi les stratégies qui augmentent l'économie intrinsèque de tout mot ou terme. Ces effacements réduisent, en effet, la quantité de travail que coûte normalement l'emploi d'un terme syntagmatique à $n$ constituants, sans nuire toutefois à la fonction dénominative de ces unités du lexique, vu que le sémantisme du terme est intégralement transféré à sa forme plus courte. Outre cette économie de travail, conséquence directe de la linéarité de la langue, les effacements neutralisent, de plus, les effets secondaires souvent fâcheux qui découlent de la réitération du terme. Ils rétablissent, en effet, le poids informatif du terme quand celui-ci est employé de façon répétitive.

Du point de vue du décodage, les effacements, tant lexicogènes que non lexicogènes, participent à la construction de la structure cohésive du texte. Le texte dérive de cette structure son caractère unitaire, c'est-à-dire sa textualité caractéristique, qui détermine grandement la facilité de sa lecture, si on ne tient pas compte bien évidemment des connaissances extralinguistiques nécessaires à son interprétation. Par le biais des effacements qui assurent le transfert du sens du terme à ses formes réduites, le terme réitéré parvient, en effet, à établir des relations cohésives entre les propositions et les phrases qui contiennent sa forme pleine et ses formes réduites. Par exemple, dans les trois extraits analysés précédemment, il existe, en vertu de l'identité de leur sens, un rapport cohésif entre les membres de chacune des trois paires réductionnelles. Dans le premier extrait, le rapport est de type anaphorique, la désambiguïsation de la forme non lexicogène, orbite, nécessitant le concours de orbite des satellites géostationnaires, forme qui précède orbite dans la phrase. Dans le deuxième extrait, le rapport peut être qualifié de cataphorique, étant donné que la forme pleine, orbite des satellites géostationnaires, suit la forme plus courte, orbite géostationnaire. Enfin, dans le dernier extrait, le rapport est à nouveau anaphorique, la forme courte, élément, suivant la forme complète du terme, élément de batterie. Ces relations cohésives font transiter le sens, celui du terme en l'occurrence, d'une proposition à l'autre et d'une phrase à l'autre du texte et créent ainsi un tout qui se tient. 
En somme, les effacements tant lexicogènes que non lexicogènes effectués par la réduction assurent l'efficacité du texte en tant que véhicule de connaissances d'au moins deux façons. En assurant l'équilibrage des deux efforts d'encodage et de décodage, ils s'opposent, d'une part, à l'érosion du contenu informatif du terme quand celui-ci est réitéré, et contribuent, de l'autre, à la construction de l'échafaudage cohésif du texte. En effet, ils permettent au terme réitéré d'agir comme un moyen cohésif, qui établit, éventuellement secondé par des moyens cohésifs grammaticaux, tel l'adjectif démonstratif, des relations cohésives entre la phrase ou la proposition, qui contient sa première occurrence, et les propositions et phrases subséquentes, qui contiennent ses transformations le plus souvent plus économiques.

Le rôle textogène des effacements maintenant établi, il nous reste à faire une dernière remarque au sujet du terme syntagmatique. Cette remarque est la suivante: le terme syntagmatique est une unité fondamentalement paradigmatique. Derrière tout terme syntagmatique se cache, en effet, un paradigme réductionnel, qui l'aide à contribuer à la cohésion du texte de spécialité. Ce paradigme regroupe, outre la forme pleine du terme, toutes les formes réduites qui peuvent être générées en discours par des effacements contextuels lexicogènes et non lexicogènes. Nous sommes d'avis, par ailleurs, que l'emploi autonome ou quasi autonome de formes réduites, spécialement lexicogènes, signale que le paradigme réductionnel peut être mémorisé par les locuteurs, c'est-à-dire intégré dans leurs connaissances de la langue.

À titre d'exemple, voici, reconstitué et représenté sous la forme d'un tableau, le paradigme réductionnel de orbite des satellites géostationnaires:

\begin{tabular}{|l|l|}
\hline TERME & $\begin{array}{l}\text { ORBITE DES SATELLITES } \\
\text { GÉOSTATIONNAIRES }\end{array}$ \\
\hline FORME PLEINE & Orbite des satellites géostationnaires \\
\hline FORME RÉDUITE LEXICOGĖNE & Orbite géostationnaire \\
\hline FORME RÉDUITE NON LEXICOGĖNE & Orbite \\
\hline
\end{tabular}

Nous estimons que le paradigme réductionnel de tout terme mériterait d'être inclus dans les entrées d'un dictionnaire spécialisé. D'une part, parce que ce paradigme renferme des informations sur le terme et le texte de spécialité susceptibles d'intéresser les langagiers, tels les traducteurs et rédacteurs techniques. D'autre part, parce que, l'inclusion du paradigme réductionnel éviterait des échecs sur le plan de la consultation de l'ouvrage de référence. En effet, comme l'a si bien dit Gouadec (1990: 59), « compte tenu de l'impossibilité de prévoir la forme à partir de laquelle s'effectuera la recherche des données, la fiche ou le dossier concernant le terme-vedette doit donner directement accès à toutes les formes répertoriées et donc à toutes les variantes de désignation ».

\section{Une nouvelle microstructure de dictionnaire spécialisé}

Dans un article examinant la complémentarité de la terminologie et de la lexicographie, Riggs (1989: 90) affirme que «lexicography has the primary aim of helping readers interpret texts, whereas terminology aims to help writers produce texts (c'est nous qui soulignons) ». Or, très peu de produits terminologiques se conforment actuellement à cette caractérisation de la terminologie. 
Les banques de données terminologiques, fortes des progrès réalisés en informatique, ajoutent peu à peu aux rubriques traditionnelles de la fiche de terminologie, correspondant au terme-vedette, au domaine, à la définition et au contexte, de nouvelles rubriques consacrées, par exemple, aux pantonymes (termes génériques), aux idionymes (termes spécifiques) et aux isonymes (termes de même niveau) du termevedette afin de mieux situer ce dernier dans le réseau de termes dont il fait partie. Certains terminologues, tels Gouadec (1990) et Sager (1990), jugent judicieux en outre d'inclure davantage de renseignements portant sur l'emploi du terme en discours. Ainsi, Gouadec (1990: 55) propose une rubrique consacrée aux «stéréotypes phraséologiques", c'est-à-dire "[l'] ensemble des combinaisons stéréotypées dans lesquelles entre le terme-vedette». Ce terminologue argumente même qu'à la citation de contextes, héritage selon lui de la tradition lexicographique, il conviendrait de «préférer les contextes denses que sont les stéréotypes phraséologiques» (Gouadec 1990: 47). Sager (1990: 142), pour sa part, estime que «the linguistic behaviour of terms should be documented by suitable contexts so that all relevant textual variants are covered». Cependant, tout habitué des banques de terminologie sait que ces renseignements se rapportant à la mise en discours du terme constituent toujours une denrée rare.

Quant à la microstructure des dictionnaires spécialisés monolingues - ouvrages qui relèvent du fait de leur thématique de la terminologie/terminographie ou, si on préfère, de la lexicographie spécialisée, genre hybride se rapprochant de la terminographie (Gaudin 1995: 11) -, sa physionomie demeure fondamentalement inchangée malgré la refonte aussi prudente soit-elle de sa contrepartie informatisée. En effet, aux rubriques traditionnelles, qui renferment, en accord avec le primat accordé à la dimension notionnelle, essentiellement le terme-vedette et sa définition et plus rarement des synonymes, ne se sont jointes peu ou pas de rubriques renseignant sur l'emploi du terme, en dépit des plaidoyers en faveur de telles rubriques en terminologie.

Monolingual specialized dictionaries subdivide into general specialized dictionaries and subject-specific specialized dictionaries [...]. The organization of both types of specialized dictionary and the content of their entries are broadly similar. The headword of each entry consists of a single word or multiword unit. Each entry contains a definition which may contain cross-references to other entries. [...] Entries are unlikely to contain $[\ldots]$ any examples of usage (Pearson 1998: 70-71).

Quelques statistiques se rapportant aux textes de spécialité et aux termes suffisent, toutefois, pour souligner l'intérêt que représente très certainement l'inclusion de rubriques consacrées à la mise en discours du terme, particulièrement du terme syntagmatique, pour les traducteurs ou rédacteurs, les utilisateurs principaux de dictionnaires spécialisés. Par exemple, on estime que les textes de spécialité représentent plus de $60 \%$ de la production écrite en français (Cajolet-Laganière et Martel 1994: 269). Une analyse de corpus élémentaire effectuée par Cajolet-Laganière et Martel (1994) signale de plus que ces textes contiennent en moyenne $38 \%$ de termes simples et $72 \%$ de termes syntagmatiques. Les termes syntagmatiques, souvent majoritaires dans les textes de spécialité, le sont aussi dans les domaines du savoir. En effet, les langues de spécialité contiendraient environ $85 \%$ de termes syntagmatiques (Boulanger 1994: 261). À la lumière de ces chiffres fort impressionnants, nul ne peut contester, à notre avis, l'importance d'inclure au moins parmi les rubriques qui modèlent la microstructure d'un dictionnaire spécialisé non seulement une rubrique 
renfermant les «stéréotypes phraséologiques» du terme, comme le propose entre autres Pearson (1998), mais aussi une rubrique listant les formes réduites par le biais desquelles ces termes participent à l'organisation de la cohésion du texte de spécialité. La surabondance de termes syntagmatiques dans les domaines du savoir et de ce fait dans les textes de spécialité laisse d'ailleurs entrevoir l'ampleur probable du phénomène de la réduction, bien que nous ne disposions pas encore de statistiques à ce sujet au moment d'écrire cet article.

Sous 3.1 ci-dessous, nous présenterons la physionomie de la rubrique consacrée au paradigme réductionnel, et sous 3.2, pour terminer, nous montrerons comment elle peut être intégrée à une entrée possédant une microstructure élémentaire.

\subsection{Contenu et forme de la rubrique du paradigme réductionnel}

La rubrique du paradigme réductionnel contient toutes les formes du terme syntagmatique, excepté sa forme pleine. Cette dernière se place dans la rubrique du terme-vedette. Quant aux formes réduites, nous jugeons important de lister les formes lexicogènes investies d'une autonomie considérable aussi bien que les formes non lexicogènes le plus souvent dépourvues d'autonomie, bien que nous soyons consciente que cette décision de notre part risque de soulever des critiques. Toutefois, l'inclusion des formes non lexicogènes permet de déboucher sur une rubrique exhaustive qui représente le paradigme réductionnel complet du terme, à l'exclusion cependant des sigles et acronymes qui relèvent d'une autre opération mais dont l'inclusion dans la rubrique est tout à fait envisageable.

La rubrique, quant à elle, distingue nettement entre les formes lexicogènes et les formes non lexicogènes. Elle se divise, en effet, en deux sections, l'une listant les formes lexicogènes et l'autre les formes non lexicogènes. Afin de souligner la plus grande autonomie des formes lexicogènes, la rubrique les mentionne avant les formes non lexicogènes. Elle accole, de plus, aux deux sortes de formes réduites un code signalant leur nature: RL pour les formes lexicogènes et RC pour les formes non lexicogènes. Il va sans dire que la valeur de ces codes devra être expliquée dans la préface du dictionnaire recourant à cette rubrique. L'inclusion des sigles et acronymes nécessiterait l'ajout d'une troisième section suivant celle des formes non lexicogènes afin de ne pas briser la continuité du paradigme réductionnel proprement dit. Enfin, dans leurs sections respectives, la rubrique classe les formes lexicogènes et non lexicogènes par ordre alphabétique et selon le principe d'économie du langage, c'est dire que, du point de vue de la longueur, l'ordre de présentation est régressif, les formes plus longues et donc plus complètes précédant les formes plus courtes.

Voici trois exemples, le premier représentant le paradigme réductionnel plutôt riche de terminal mobile terrestre, le deuxième le paradigme moyen de moteur à propergol liquide, et le troisième celui plutôt restreint de élément de batterie.

Rubrique du paradigme réductionnel de terminal mobile terrestre:

mobile terrestre $_{\mathrm{RL}}$

terminal mobile RL $_{2}$

mobile $_{\mathrm{RL}}$

terminal $_{\mathrm{RC}}$ 
Rubrique du paradigme réductionnel de moteur à propergol liquide:

moteur liquide $_{\mathrm{RL}}$

moteur $_{\mathrm{RC}}$

Rubrique du paradigme réductionnel de élément de batterie:

élément ${ }_{\mathrm{RC}}$

Il n'est pas inutile de remarquer que la structure de cette rubrique ne convient non seulement aux termes syntagmatiques français, mais aussi à leurs homologues d'autres langues, tel l'anglais, qui sont également peu figés et se comportent de ce fait d'une façon similaire en discours. Ainsi, on peut opposer au paradigme réductionnel de moteur à propergol liquide celui plus riche de son équivalent anglais, liquid-fuel rocket engine.

Rubrique du paradigme réductionnel de liquid-fuel rocket engine:

liquid-fuel engine ${ }_{\mathrm{RL}}$

liquid-fuel rocket ${ }_{\mathrm{RL}}$

liquid engine ${ }_{\mathrm{RL}}$

rocket engine $\mathrm{RC}_{\mathrm{RC}}$

engine $_{\mathrm{RC}}$

On voit donc que la rubrique que nous proposons aura sa place également dans les dictionnaires spécialisés bilingues.

\subsection{Place de la rubrique du paradigme réductionnel dans une microstructure élémentaire}

Dans une microstructure de dictionnaire élémentaire se préoccupant essentiellement de la définition du terme-vedette, il est préférable de placer la rubrique du paradigme réductionnel immédiatement en dessous du terme-vedette, vu que les formes réduites sont des variantes formelles de ce dernier, mais avant la définition, étant donné que cette dernière vaut pour toutes les formes, pleine et réduites, du termevedette. La microstructure élémentaire:

\section{TERME-VEDETTE \\ formes réduites $_{\mathrm{RL}}$ \\ formes réduites ${ }_{\mathrm{RC}}$}

\section{Définition}

est celle qui a été retenue pour les deux entrées dictionnairiques ${ }^{7}$ ci-dessous que nous offrons à titre d'exemple.

Entrée dictionnairique 1:

ANTENNE EN RÉSEAU

antenne réseau $_{\mathrm{RL}}$

réseau $\mathrm{RL}_{\mathrm{L}}$

antenne $_{\mathrm{RC}}$

Antenne constituée par un ensemble d'éléments rayonnants identiques, se déduisant l'un de l'autre par translation, et alimentés par une même source, chacun avec une amplitude et une phase qui dépendent de sa position, de manière à obtenir une surface caractéristique de rayonnement donnée. 
Entrée dictionnairique 2:

ANTENNE EN RÉSEAU PLAN

antenne réseau plan ${ }_{\mathrm{RL}}$

réseau plan ${ }_{\mathrm{RL}}$

réseau ${ }_{\mathrm{RC}}$

antenne $_{\mathrm{RC}}$

Antenne en réseau dont les éléments rayonnants se déduisent l'un de l'autre par des translations dans un plan.

Si le terme-vedette possède des synonymes et si la microstructure élémentaire comprend une rubrique consacrée aux synonymes, celle-ci se placera préférablement en dessous de la définition afin de bien distinguer le terme-vedette et les formes qu'il peut revêtir en discours de ses synonymes susceptibles de se transformer eux aussi en discours. Nous obtenons dans ce cas la microstructure suivante:

TERME-VEDETTE

formes réduites ${ }_{\mathrm{RL}}$

formes réduites $\mathrm{RC}_{\mathrm{RC}}$

Définition

Syn.: termes synonymes

Voici une entrée dictionnairique ${ }^{8}$ ayant cette microstructure élargie:

\section{ANTENNE À COUVERTURE GLOBALE}

antenne globale $_{\mathrm{RL}}$

antenne $_{\mathrm{RC}}$

Antenne de satellite de télécommunications dont le faisceau couvre la totalité du globe terrestre.

Syn. : antenne à faisceau global

Au sujet des synonymes, il nous faut remarquer que nous jugeons préférable de les traiter dans des entrées séparées à la façon d'un dictionnaire de langue. L'approche sémasiologique, allant du terme au sens et non du sens au terme, nous semble en effet plus compatible, du point de vue idéologique, avec ce que la microstructure que nous préconisons cherche à accomplir: refléter la variation en discours du terme syntagmatique. Le terme antenne à faisceau global aura par conséquent sa propre entrée, qui renfermera le paradigme réductionnel de ce terme. Outre cet avantage idéologique, le traitement séparé des synonymes permettra par ailleurs une analyse plus fine en cas de quasi-synonymie et évitera que les entrées ne deviennent trop chargées de renseignements se rapportant en fait à des termes différents.

Enfin, si la microstructure, en accord avec les souhaits des utilisateurs, renferme une rubrique listant les "stéréotypes phraséologiques", cette rubrique se placera avant celle des synonymes, car elle ne concerne que le terme-vedette ainsi que ses formes réduites. Cette nouvelle microstructure prend alors la forme suivante: 
TERME-VEDETTE

formes réduites ${ }_{\mathrm{RL}}$

formes réduites $_{\mathrm{RC}}$

Définition

Phras.: stéréotypes phraséologiques

Syn. : termes synonymes

Si nous ajoutons cette rubrique à l'entrée précédente, nous obtenons ce qui suit:

ANTENNE À COUVERTURE GLOBALE

antenne globale ${ }_{\mathrm{RL}}$

antenne $_{\mathrm{RC}}$

Antenne de satellite de télécommunications dont le faisceau couvre la totalité du globe terrestre.

Phras. : l'antenne à couverture globale couvre (la surface visible de la Terre) ${ }^{9}$

Syn. : antenne à faisceau global

Il va sans dire que cette nouvelle microstructure convient également à la description des termes d'autres langues, tel l'anglais, dont le comportement en discours se rapproche de celui des termes français. Ainsi, si nous intégrons le paradigme réductionnel de liquid-fuel rocket engine dans cette dernière microstructure qui liste également les stéréotypes phraséologiques et les synonymes du terme-vedette, nous obtenons l'entrée ${ }^{10}$ suivante:

\section{LIQUID-FUEL ROCKET ENGINE}

liquid-fuel engine ${ }_{\mathrm{RL}}$

liquid-fuel rocket ${ }_{\mathrm{RL}}$

liquid engine $\mathrm{RL}_{\mathrm{RL}}$

rocket engine $e_{\mathrm{RC}}$

engine $_{\mathrm{RC}}$

Class of rocket engines using liquid combustibles which are contained in tanks and are fed into the thrust chamber through an injector head by a propellant supply system.

Phras. : the thrust provided by a liquid-fuel rocket engine

Syn. : liquid-propellant rocket engine; liquid-propellant rocket motor

Pour conclure ce sous-point, il nous faut mentionner qu'il conviendrait d'ajouter un index alphabétique au dictionnaire qui incorpore dans sa microstructure la rubrique du paradigme réductionnel. Cet index listera tous les termes contenus dans le dictionnaire ainsi que toutes les formes réduites lexicogènes. Quant aux formes réduites non lexicogènes, elles n'ont pas besoin d'y figurer. L'inclusion des formes non lexicogènes alourdirait indûment l'index d'items identiques, dont un seul constituerait un terme à proprement parler, par exemple antenne, et les autres du type antenne $_{R C}$ des renvois à des entrées dont le terme-vedette commencerait par antenne. Le dictionnaire permettra ainsi, comme de coutume, une consultation traditionnelle basée sur l'ordre alphabétique des entrées, mais comportera, sous la forme de l'index, 
un outil de recherche supplémentaire visant à diminuer les échecs de consultation quand celle-ci se fait à partir d'une forme qui n'est pas la forme canonique du terme.

\section{Conclusion}

Dans cet article, nous avons proposé d'inclure dans la microstructure d'un dictionnaire spécialisé monolingue une nouvelle rubrique consacrée au paradigme réductionnel du terme syntagmatique. L'élaboration de cette rubrique s'inscrit dans un projet de recherche plus vaste qui étudie l'interaction du terme, spécialement du terme syntagmatique, avec le texte de spécialité. Nous nous sommes basée sur quelques-uns des résultats de ce projet de recherche, notamment ceux ayant trait à la façon dont le terme syntagmatique contribue par le biais de la réduction à la cohésion du texte de spécialité, afin de cerner le statut des formes réduites et d'étayer le bien-fondé de leur inclusion dans un dictionnaire spécialisé. La rubrique qui a fait l'objet de cet article constitue d'ailleurs le premier pas vers la conception d'un dictionnaire spécialisé, destiné aux traducteurs et rédacteurs techniques, qui incorporera davantage de rubriques décrivant la mise en discours du terme. Il s'agit d'un dictionnaire qui se voudra sémasiologique, mais qui accordera toujours une extrême importance au notionnel (définition, identification éventuelle des pantonymes, idionymes et isonymes), toutefois, tout en donnant au linguistique, à l'usage, toute sa place.

\section{NOTES}

1. Cet article est basé sur une communication faite par nous au seizième colloque annuel de l'Association canadienne de traductologie (ACT) tenu à l'Université Dalhousie du 29 au 31 mai 2003. L'article a grandement bénéficié des remarques pertinentes des membres de l'assistance, dont Sylvie Vandaele.

2. Par «dictionnaire spécialisé », nous n’entendons dans le présent article que les dictionnaires spécialisés monolingues.

3. Les dictionnaires spécialisés ayant fait l'objet de l'enquête du Centre de Terminologie de Bruxelles étaient pour la plupart du type multilingue et non monolingue. Cependant, faute d'une enquête consacrée spécifiquement aux dictionnaires monolingues, nous nous sommes permis cette extrapolation car, comme leurs homologues multilingues, les dictionnaires spécialisés monolingues fournissent également très peu d'informations sur l'usage des termes qu'ils définissent.

4. Pour de plus amples renseignements sur la réduction et sur les règles qui la gouvernent en discours, nous renvoyons le lecteur à Collet (2003), article qui décrit les composantes principales d'une grammaire descriptive de la réduction.

5. Dans les extraits illustrant la réduction, nous mettons en gras les formes des termes syntagmatiques dont la linéarité est modifiée par une réduction.

6. Pour de plus amples renseignements sur les propriétés textogènes de la réduction, nous renvoyons le lecteur à Collet (2000).

7. Les définitions dans les deux entrées dictionnairiques ont été tirées de la norme française NF C01-062.

8. La définition de cette entrée provient de Nouvel, Marcel (1983): Dictionnaire bilingue des télécommunications internationales. 1. Théorie des antennes, Montréal, Téléglobe Canada, 128 p.

9. Le contenu de la rubrique des «stéréotypes phraséologiques» dans cette entrée ainsi que dans l'entrée suivante est provisoire et n'a été fourni qu'à titre d'exemple pour les besoins de la démonstration.

10. La définition de liquid-fuel rocket engine est basée sur celle fournie par The New Encyclopoedia Britannica mais a été adaptée par nous. 


\section{RÉFÉRENCES}

Boulanger, J.-C. (1994): «L’aménagement du lexique spécialisé dans le dictionnaire de langue. Du prélexicographique au microstructurel», dans: Langues et sociétés en contact. Mélanges offerts à Jean-Claude Corbeil, P. Martel et J. Maurais (dir.), Tübingen, Max Niemeyer Verlag, p. 253-268.

Brillaud, J.-P. (1988) : «Télécommunications par satellite», Techniques de l’ingénieur, E7 II Électronique - Télécommunications, p. 570-1 - 570-11.

Cajolet-Laganière, H. et P. Martel (1994) : «Caractéristiques lexicales des textes scientifiques: premières analyses», dans: Langues et sociétés en contact. Mélanges offerts à Jean-Claude Corbeil, P. Martel et J. Maurais (dir.), Tübingen, Max Niemeyer Verlag, p. 269-282.

Collet, T. (2000): La réduction des unités terminologiques complexes de type syntagmatique, thèse de doctorat, Université de Montréal.

Collet, T. (2003, à paraitre): «A Two-Level Grammar of the Reduction Processes of French Complex Terms in Discourse», Terminology, 9-1.

Dancette, J. (1995): «Organisation conceptuelle du domaine et structure de dictionnaire L'exemple du commerce de détail», TTR - Traduction, Terminologie, Rédaction, VIII (2), p. 151-174.

De Beaugrande, R.-A. and W. U. Dressler (1986): Introduction to Text Linguistics, London/ New York, Longman.

Fitamant, H. (1971): «L'alimentation électrique des satellites», L'Aéronautique et l'Astronautique, 7, p. 19-30.

Gaudin, F. (1995): «Dire les sciences et décrire les sens: entre vulgarisation et lexicographie, le cas des dictionnaires de sciences», TTR - Traduction, Terminologie, Rédaction, VIII (2), p. 11-27.

Gouddec, D. (1990): Terminologie. Constitution des données, Paris, AFNOR.

Hermans, A. et C. De Schaetzen (1991): «Étude fonctionnelle des dictionnaires techniques par les méthodes de l'analyse de la valeur», Le Langage et l'Homme, XXVI (4), p. 301-303.

Kocourek, R. (1991): La langue française de la technique et de la science. Vers une linguistique de la langue savante, $2^{\mathrm{e}}$ édition, Wiesbaden, Oscar Brandstetter Verlag.

Pearson, J. (1998): Terms in Context, Amsterdam/Philadelphia, John Benjamins Publishing Company.

RIGGS, F. (1989) : «Terminology and Lexicography: their Complementarity», International Journal of Lexicography, 2-2, p. 89-110.

SAgER, J. C. (1984) : «Terminology and the Technical Dictionary», in: LEXeter '83 Proceedings. Papers from the International Conference on Lexicography at Exeter, 9-12 September 1983, R. R. K. Hartmann (Ed.), Tübingen, Max Niemeyer Verlag, p. 315-326.

SAger, J. C. (1990): A Practical Course in Terminology Processing, Amsterdam/Philadelphia, John Benjamins Publishing Company.

Slodzian, M. (1997) : «Un obstacle à la conception de nouveaux outils: l'approche discontinue et fragmentaire de l'objet "dictionnaire" ", dans: Les dictionnaires spécialisés et l'analyse de la valeur, A. Hermans (dir.), Louvain-La-Neuve, Peeters, p. 23-31.

ZIPF, G. K. (1965): Human Behavior and the Principle of Least Effort. An Introduction to Human Ecology, New York/London, Hafner Publishing Company. 